\title{
Flow Rate Control in Pressure- Programmed Capillary Supercritical Fluid Chromatography
}

\author{
Hans-Gerd Janssen, * Jacques A. Rijks, and Carl A. Cramers \\ Eindhoven University of Technology, Laboratory of Instrumental Analysis, \\ P.O. Box 513, $5600 \mathrm{MB}$ Eindhoven, The Netherlands
}

\begin{abstract}
A versatile and simple system is described that allows variation of the column flow rate in open-tubular capillary supercritical fluid chromatography using both on-column and postcolumn detection. The system is based on column-effluent splitting in a low-dead-volume T piece at the column exit just before detection. The flow is split into two streams. One stream is fed to a flame ionization detector, and the second is used to control the overall mass flow through the column. Flow through linear restrictors is studied as a function of the pressure drop over the restrictor at ambient temperature. We derived a simple relationship from this study that quantifies the flow rate characteristics of the dual-restrictor system as a function of the column pressure and the restrictor back pressure.

The variable-flow system was employed to control independently column pressure and mass flow rate in pressure-programmed analysis. Resolution in flow-controlled pressure-programmed elution was shown to be superior to that achieved in fixed-restrictor systems. Furthermore, the applicability of the system as a convenient means of measuring Van Deemter curves and diffusion coefficients is demonstrated.
\end{abstract}

Key words: supercritical fluid chromatography ( $S F C$ ), pressure-programmed analysis, mass flow rate control, Van Deemter curves, diffusion coefficients

\section{INTRODUCTION}

The use of supercritical fluids as mobile phases in chromatography offers several important advantages over gases or liquids. The ability to separate nonvolatile or thermally labile components at mild temperatures was recognized as a distinct advantage over gas chromatography (GC) (1). Compared with liquid chromatography (LC), supercritical fluid chromatography (SFC) offers promise because it is much more compatible with various detection systems (2).

Retention in SFC is a complex function of temperature, mobile phase composition, and, especially, pressure (and, hence, density). For the optimization of resolution, the continuous programming of at least one of these parameters is required in most situations. Pressure/density gradients, composition gradients, positive- or negative-temperature programming, and multiple gradient separations have been demonstrated with both open-tubular and packed-column SFC
(3). Although the main goal of programming techniques is to reduce retention, the final result, in terms of abilities to achieve high resolution and elute high molecular weight compounds, depends on the programming technique chosen. Schmitz et al. compared composition-programmed packed-column SFC with pressure-programmed open-tubular SFC and found that composition gradients seemed to be better suited for the elution of oligomeric series of high molecular weight compounds (4). Takeuchi compared negative-temperature programming and pressure programming in the separation of Triton X-100 and Nonidet P40 by capillary SFC (5). The resolution obtained with negative-temperature programming was comparable to or slightly poorer than that achieved by pressure programming.

The ability to alter the solvent strength of the mobile phase by adjusting an instrumental parameter, the pressure, enhances

*Author to whom correspondence should be addressed

(C) 1990 Aster Publishing Corporation 


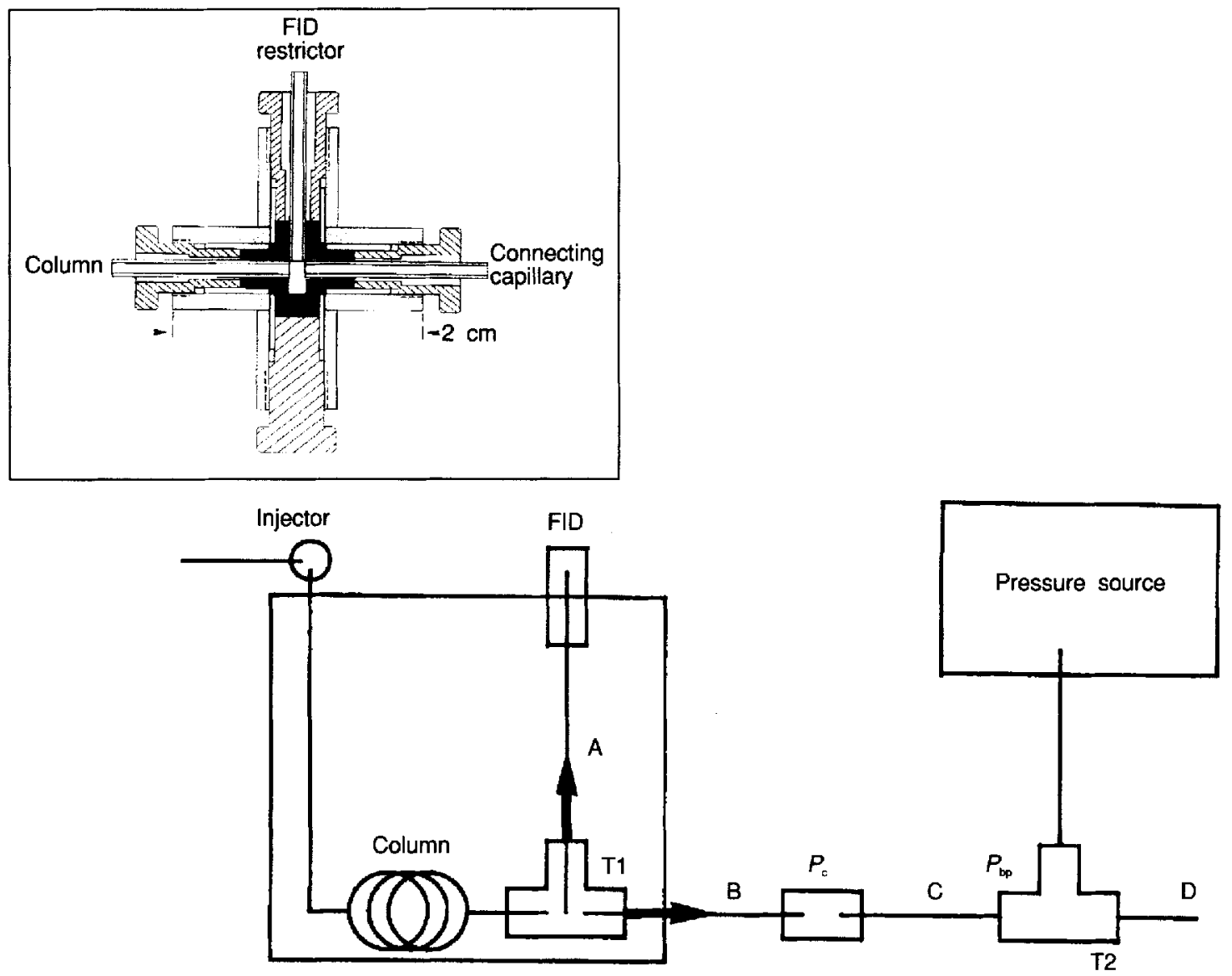

Figure 1. Experimental setup (for discussion, see text). $A=13 \mathrm{~cm} \times 4.6 \mu \mathrm{m}$ FID restrictor, $B=30 \mathrm{~cm} \times 75 \mu \mathrm{m}$ connecting capillary, $C=52 \mathrm{~cm} \times 12.6 \mu \mathrm{m}$ variable-flow restrictor, and $D=25 \mathrm{~cm} \times 12.6 \mu \mathrm{m}$ vent restrictor. The inset shows an enlargement of the $T$ piece $T 1$.

the attractiveness of supercritical fluids as mobile phases. The simple instrumental requirements have made pressure/density programming the most frequently employed programming technique. Increasing the mobile phase pressure, however, not only affects the solvent strength of the mobile phase, but also entails considerable changes in the viscosity, diffusivity, and selectivity of the mobile phase. In addition, the properties of the stationary phase can change if increasing amounts of mobile phase are dissolved in the stationary phase during programming (6). The analytes eluting at higher pressures have smaller diffusion coefficients, both because of their larger size and because of the increased density of the mobile phase. To keep the efficiency constant, the linear velocity should be appreciably reduced. However, in chromatographic systems equipped with a fixed restrictor, the mass flow rate cannot be controlled. Due to an increase of the pressure drop over the restrictor during pressure programming, the mass flow rate increases. In this situation, programming of the column pressure usually is accompanied by an increasing linear velocity in the chromatographic column. As a result, a decreased resolution at higher densities often is observed. Systems designed to independently control pressure and velocity have been described for both packed and capillary SFC (7-9). These systems, however, are not applicable to situations in which a postrestrictor detection system such as a flame ionization detector (FID) or a mass spectrometer (MS) is used. Recently, Raynie et al. described the use of a backpressure-regulated restrictor for flow control in capillary SFC based on a sheath nozzle previously developed for supersonic jet spectroscopy (10). Although it was suggested that this system would be applicable to systems using postrestrictor detection, the authors did not demonstrate the interfacing with this type of detector.

In this paper, a method is described that allows independent control of column pressure and linear velocity in capillary SFC using either high- or low-pressure detection. The system is employed to enhance separation quality in the latter part of a pressure-programmed analysis. Furthermore, it can be used to scout rapidly for optimum pressures and temperatures in the design of SFC separations. The applicability of the system 
as a convenient means of measuring Van Deemter curves and diffusion coefficients under SFC conditions is demonstrated.

\section{INSTRUMENTATION}

The chromatographic system utilized a modified Varian 8500 syringe pump (Varian Associates, Sunnyvale, Califomia, USA) that was under computer control to generate a pulsefree flow of mobile phase. Sample introduction was accomplished at ambient temperature using a pneumatically actuated injection valve (model A-3-Ni4W, VICI AG, Schenkon, Switzerland) equipped with a $0.04 / 0.06 \mu \mathrm{L}$ rotor. The injector was used in the timed-split mode without subsequent flow splitting. Temperature control and flame ionization detection were provided by a Varian 3300 gas chromatograph. The mobile phase used in the experiments was $99.996 \%$ carbon dioxide (Intermar B.V., Breda, The Netherlands). The chromatographic column was a $8.2 \mathrm{~m} \times 107 \mu \mathrm{m}$ i.d. OV-1 capillary (Rescom, Kortrijk, Belgium). The experimental setup is shown in Figure 1.

The outlet of the column was connected to a specially designed low-dead-volume T piece (T1) (Gerstel GmbH, Mülheim a/d Ruhr, FRG). A detailed schematic of $T$ piece $T 1$ is given in the enlargement in Figure 1. The $T$ piece consists of a cross-piece with one blind nut. The ferrules are made of a cartridge containing graphite as the sealing material. The bore diameter of the piece was $270 \mu \mathrm{m}$, which was matched closely to the outside diameter of the capillaries. The total volume of the piece was less than $80 \mathrm{~nL}$. No adverse effect of the $T$ piece on the efficiency of the system was observed. One branch of the $T$ was a $13 \mathrm{~cm} \times 4.6 \mu \mathrm{m}$ i.d. fused-silica capillary (capillary A), which was directly installed in the FID. The FID was heated to $340^{\circ} \mathrm{C}$. The other branch was a $30 \mathrm{~cm}$ $\times 75 \mu \mathrm{m}$ i.d. fused-silica capillary (B). Outside the GC oven, this capillary was coupled with a $52 \mathrm{~cm} \times 12.6 \mu \mathrm{m}$ i.d. restrictor (C) using a low-dead-volume coupling piece (Gerstel $\mathrm{GmbH}$ ). Flow rate control through this branch was accomplished by regulating a back pressure of either helium supplied by a high-pressure regulator or carbon dioxide supplied by a second Varian 8500 syringe pump, which was modified for constant pressure or pressure-programmed operation. To avoid pressure buildup at the outlet of the flow restrictor, a vent restrictor (D) was coupled with the control line using a second $\mathrm{T}$ piece (T2).

The column effluent is split into two streams. One stream is fed to the FID, and the second is used to control the total flow rate through the column. The column flow rate is determined by the sum of the flow rate through the FID (restrictor A) and the flow rate through the variable-flow restrictor (C). Control of the column flow rate is achieved by manipulating the flow rate through the variable restrictor. Because only part of the column effluent is used for detection, the absolute sensitivity of the system is reduced. Restrictor dimensions were chosen empirically to give a flow rate ratio of approximately 1:4 between the FID and the variable restrictor, while maintaining the desired total flow rate. Linear restrictors were chosen because they enable easy flow rate adjustment. Other restrictor types such as integral or tapered restrictors, which are known to give better chromatographic performance, can also be used, as will be discussed later.

The column temperature was $120^{\circ} \mathrm{C}$ throughout the experiments. Mobile phase linear velocities were determined by injecting methane as an unretained component.

\section{THEORY}

In order to describe the mass flow rate through the chromatographic column, we studied the flow rates through linear restrictors at various inlet and outlet pressures. The total flow rate through the column is the sum of the flow rates through the detector and the variable restrictor. The total flow rate can be quantified when the two individual contributions are known. For flow rates through linear and tapered restrictors, Bally has shown that the mass flow rate at high temperatures and atmospheric outlet pressure is proportional to the column pressure (11). Based on his results, the mass flow rate through the FID restrictor, $F_{\mathrm{Fid}}$, can be described by equation 1:

$$
F_{\mathrm{Fid}}=a P_{\mathrm{c}}+b
$$

where $P_{\mathrm{c}}$ is the column pressure and $a$ and $b$ are constants that depend on the restrictor dimensions and the temperature.

Quantification of the flow rate through the variable-flow restrictor can be achieved by assuming that the effluent possesses either liquid- or (ideal) gaslike behavior in the restrictor. With high pressure at the restrictor outlet and ambient temperature, more liquidlike conditions prevail in the flow restrictor ("C" in Figure 1). In this situation, the mass flow rate in a linear flow restrictor, $F_{\mathrm{fr}}$, can be approximated by the Poiseuille equation for laminar flow:

$$
F_{\mathrm{fr}}=\left[\left(\pi \rho d^{4}\right) /(128 \eta L)\right]\left(P_{\mathrm{c}}-P_{\mathrm{hp}}\right)
$$

where

$\rho=$ fluid density, $\eta=$ viscosity, $L=$ restrictor length, $d=$ restrictor diameter, $P_{\mathrm{c}}=$ column pressure, and $P_{\mathrm{bp}}=$ back pressure applied to the restrictor. For ideal liquids, both $\rho$ and $\eta$ are independent of the pressure. For carbon dioxide at ambient temperature, both parameters are affected by pressure changes, but the ratio of density to viscosity remains almost constant in the liquid region. Equation 2 shows that when the mass flow rate through the variable-flow restrictor is plotted against the pressure drop over the restrictor, a straight line should result. Equation 2 assumes that the outlet pressure of the variable-flow restrictor equals the back pressure applied by the high-pressure device. This assumption will only be correct if the back pressure is higher than the exit pressure at sonic outlet conditions. It was shown by Smith et al. that incomplete expansion of the fluid in the restrictor is likely to occur, especially when one uses restrictors with low length-to-diameter ratios (12). In this case, equation 2 is invalid because 


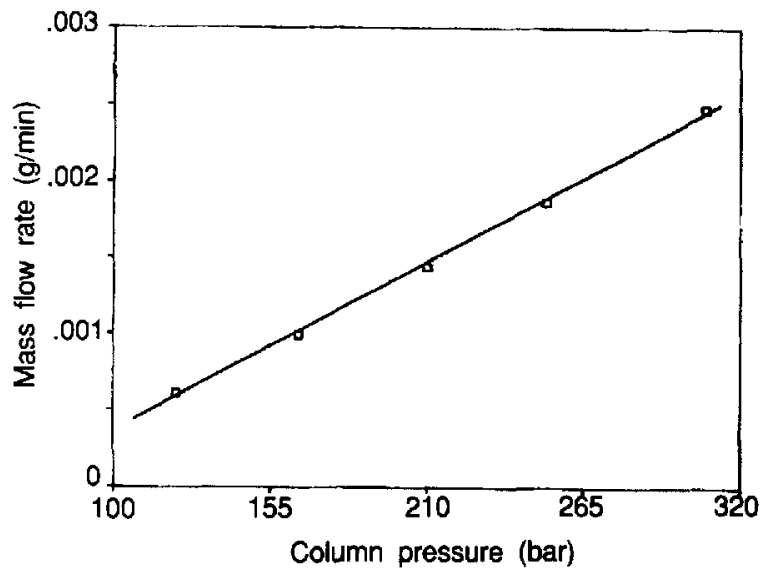

Figure 2. Mass flow rate through $13 \mathrm{~cm} \times 4.6 \mu \mathrm{m}$ FID restrictor. Variable restrictor closed. Oven temperature: $120^{\circ} \mathrm{C} ; \mathrm{FID}$ restrictor temperature: $340^{\circ} \mathrm{C}$.

the actual pressure at the restrictor outlet does not equal the back pressure.

\section{RESULTS AND DISCUSSION}

Mass flow through fixed and back-pressure-controlled restrictors. Figure 2 shows the measured mass flow rate through the column with the variable restrictor closed. Mass flow rates were obtained from the linear velocity measured by methane injections, the column cross-sectional area, and the carbon dioxide densities calculated according to the IUPAC equation of state (13).

Figure 2 shows a good agreement between the experiments and equation 1 . To test the validity of the assumptions leading to equation 2, a plot of the measured mass flow rate through the column is given in Figure 3 at different pressure drops over the variable restrictor (C). The column pressure, and hence the restrictor inlet pressure, was 260 bar.

In Figure 3, a linear relationship is observed between the measured mass flow rate and the pressure drop over the restrictor, which is in agreement with equation 2. Deviations from linearity occurred only wher the back pressure became lower than the vapor pressure of carbon dioxide at ambient temperature. In this case, a complex situation prevails in which liquid carbon dioxide evaporates in the restrictor at a position that depends on the pressure (12). After combining equations 1 and 2 , the total flow rate through the column, $F_{\text {total }}$ can be expressed by equation 3 :

$$
F_{\text {total }}=F_{\mathrm{Fid}}+F_{\mathrm{fr}}=a P_{\mathrm{c}}+b+c \Delta p
$$

where

$$
\Delta p=P_{\mathrm{c}}-P_{\mathrm{bp}}
$$

A complete quantitative characterization of the dual-restric-

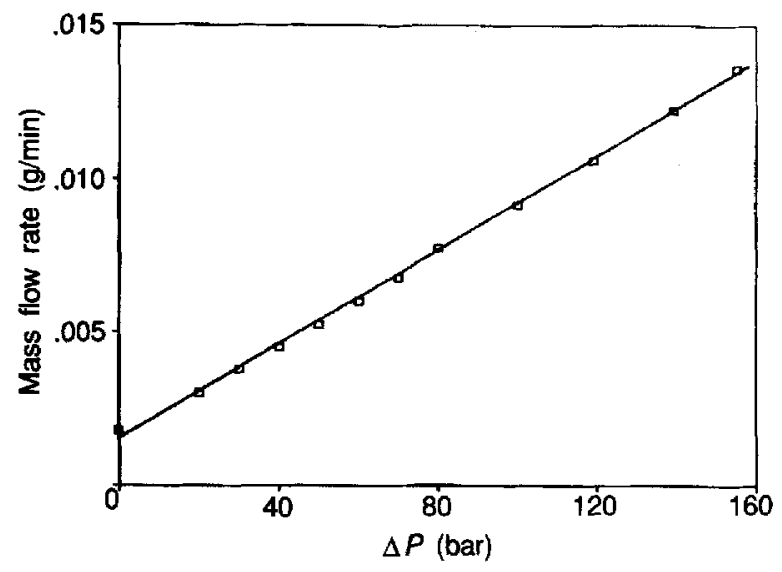

Figure 3. Column mass flow rate at different pressure drops $\Delta \mathrm{p}$ over the variable-flow restrictor. Column inlet pressure: 260 bar. Oven temperature: $120^{\circ} \mathrm{C}$. FID restrictor as in Figure 2. Variable-flow restrictor dimensions: $52 \mathrm{~cm} \times 12.6$ $\mu \mathrm{m}$; temperature $24^{\circ} \mathrm{C}$.

tor system can be obtained by measuring the three constants, two for quantifying the flow rate through the FID restrictor and one for characterizing the variable-flow restrictor.

Mass flow rate control during pressure programming. Figure 4 illustrates how the dual-restrictor system can be applied to control the linear velocity during a pressure-programmmed analysis. The upper line gives the variation of the column inlet pressure during a linear pressure-programmed capillary SFC analysis. The lower line depicts the variablerestrictor back pressure.

The pressure gradient applied to the variable-flow restrictor

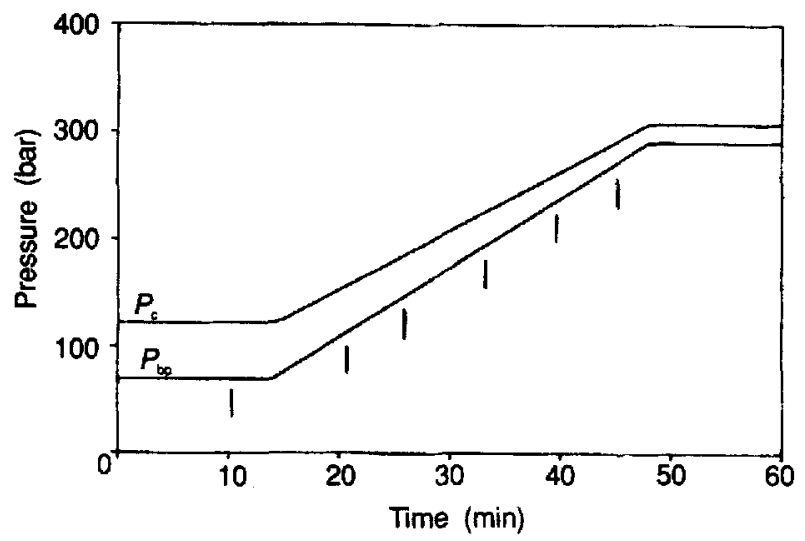

Figure 4. Column inlet pressure $\left(\mathrm{P}_{c}\right)$ and variable-restrictor back pressure $\left(\mathrm{P}_{b p}\right)$ during pressure programming with flow rate control. Column-pressure program: 120 bar $(14 \mathrm{~min}) \rightarrow$ 5 bar/min $\rightarrow 310$ bar. Variable-restrictor back-pressure program: 70 bar $(14 \mathrm{~min}) \rightarrow 6$ barimin $\rightarrow 290$ bar. Restrictor dimensions and operating conditions as in Figure 3. 


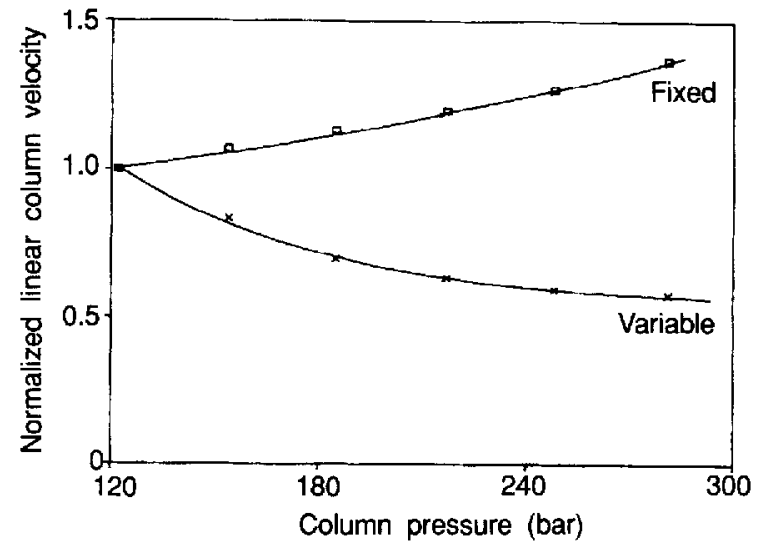

Figure 5. Linear column velocity with a fixed-and a dualrestrictor system. Restrictor dimensions and operating conditions as in Figure 3. Back-pressure program as in Figure 4.

is steeper than the gradient of the column pressure. Hence, the pressure drop over the variable restrictor decreases during the analysis. The behavior of the column flow rate, and thus the linear velocity, reflects the increasing flow rate through the FID and the decreasing flow rate through the variable restrictor. Because the absolute mass flow rate through the variable restrictor is larger than the flow rate through the FID, the overall column flow rate will decrease. During a pressure-programmed analysis, the ratio of the flow rates through the FID restrictor and the variable-flow restrictor changes. In the latter part of the analysis, a larger fraction of the column effluent is fed to the detector. This gives rise to an increasing sensitivity of the detection system, which necessitates calibration of the detector.

In Figure 5, the upper curve represents the measured, normalized linear velocity through the column in a fixedrestrictor system at different inlet pressures. The lower curve represents the normalized linear velocities using the dualrestrictor system. Experiments were performed at discrete pressures, which are indicated by the vertical lines in Figure 4. In actual pressure-programmed analysis, the linear velocities would be higher and dependent on the position in the column, owing to "compression velocity" contributions as described by Smith (14). Figure 5 clearly illustrates the ability of the dual-restrictor system to control the velocity during pressure-programmed SFC. Whereas the linear column velocity increases with pressure in a fixed-restrictor system, a decrease is observed for the dual-restrictor system. The experiments described in this work were performed with linear restrictors - that is, a linear FID restrictor and a linear variable-flow restrictor. For high molecular weight solutes, a linear FID restrictor might cause detector spiking. The experimental setup described in Figure 1, however, is not limited to linear restrictors: It also allows the use of other restrictor types for the FID. Because the performance of the variable-flow restric-

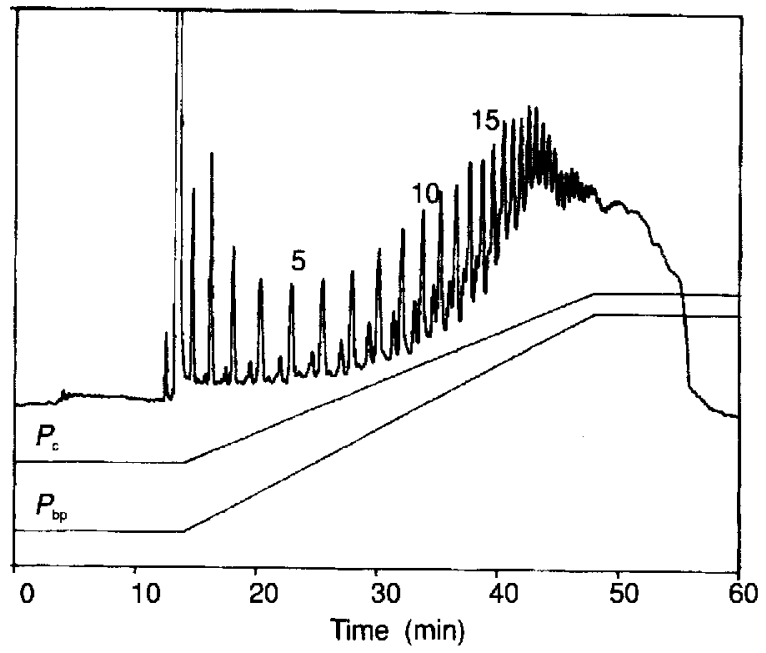

Figure 6. Analysis of poly(methylhydrosiloxane). Oven temperature: $120^{\circ} \mathrm{C}$. Restrictors as in Figure 3. Pressure programs as in Figure 4.

tor is less critical, an easily adjustable linear restrictor proves satisfactory. Figure 6 shows the analysis of a poly(methylhydrosiloxane) (PS 120, Petrarch Systems, Bristol, Pennsylvania, USA) by capillary SFC using the flowcontrolled system. Even in the flow-controlled system, an appreciable reduction of the resolution is observed in the latter part of the analysis. Here, the components in the sample almost coelute and form a hump of only partly resolved peaks. Figures $7 \mathrm{a}$ and $7 \mathrm{~b}$ show enlargements of peak pairs 9-13 for the fixed- and the variable-restrictor system, respectively. The initial linear velocities were approximately the same. The column pressure programs were identical, A better resolution is observed for the variable-restrictor system (Figure $7 \mathrm{~b}$ ). Because the linear velocity is lower in the variable-restrictor system, the elution time is longer and, hence, the elution density is higher. The higher elution density and the concomitant lower diffusivity in the mobile phase partly nullifies the gain in resolution that results from the lower linear velocity.
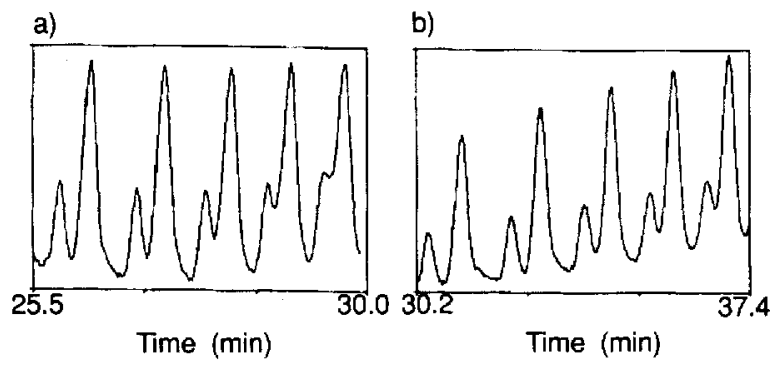

Figure 7. Comparison of the resolution with fixed-and variable-flow restrictors. a) Fixed restrictor and b) variable restrictor. Other conditions as in Figure 6. 


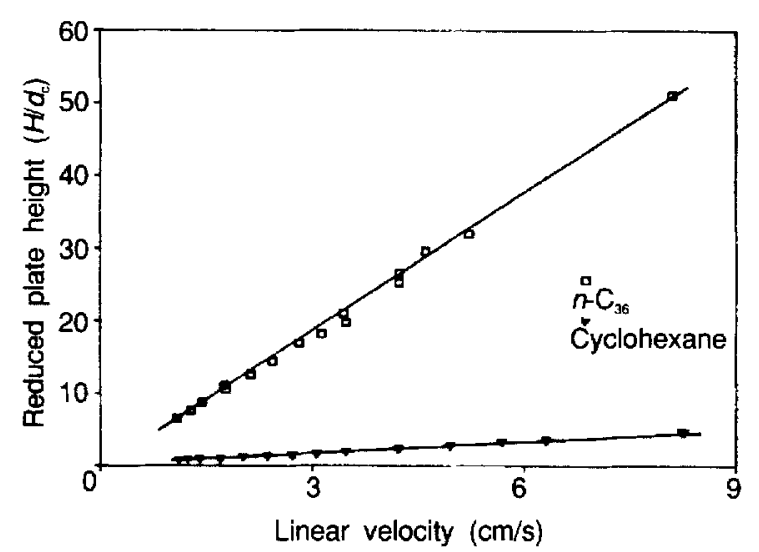

Figure 8. Reduced plate height versus linear velocity. Column pressure: 238 bar; temperature: $120^{\circ} \mathrm{C} . \mathbf{k}\left(\mathbf{n}-C_{36}\right)=1.79$; $\mathrm{k}($ cyclohexane $)=0.04$.

The improvement in resolution in the flow-controlled system would be even more pronounced if the pressure-programming rate had been adapted to the lower linear velocity.

Van Deemter curves and diffusion coefficients in capillary SFC. When the term describing the resistance to mass transport in the stationary phase is neglected, optimum linear velocity in capillary SFC is determined by the binary diffusion coefficient of the solute in the mobile phase, the column radius, and the solute capacity factor. Assuming that the pressure drop is negligible, the equation for the plate height $H$ is

$$
H=\left[\left(2 D_{\mathrm{m}}\right) / u\right]+\left[\left(f(k) d_{\mathrm{c}}^{2} u\right) / D_{\mathrm{m}}\right]
$$

where

$$
f(k)=\left(11 k^{2}+6 k+1\right) /\left[96(k+1)^{2}\right]
$$

and $D_{\mathrm{m}}=$ binary diffusion coefficient, $d_{\mathrm{c}}=$ column diameter, $u=$ linear velocity, and $k=$ capacity factor. By differentiation of equation 4 , it follows for the optimum linear velocity, $u_{\text {opt }}$;

$$
u_{\mathrm{opr}}=\left(D_{\mathrm{m}} / d_{\mathrm{c}}\right)[2 / f(k)]^{1 / 2}
$$

Because diffusion in supercritical fluids is much slower than in gases, the optimum velocity in SFC is much lower than in GC. To avoid unpractically low linear velocities, columns with small internal diameters $(<100 \mu \mathrm{m})$ are commonly used in capillary SFC. Furthermore, columns are operated at velocities far above optimum, resulting in an appreciable loss of efficiency. Linearization of equation 4 yields

$$
H u=2 D_{\mathrm{m}}+\left[\left(f(k) d_{\mathrm{c}}{ }^{2} u^{2}\right) / D_{\mathrm{m}}\right]
$$

Diffusion coefficients can be calculated either from the intercept on the $y$-axis or from the slope of a plot of $H u$ versus $u^{2}$. The fundamental aspects of the method will not be discussed because detailed reviews have been published already (15). Because $D_{\mathrm{m}}$ and $k$ are density-dependent, Van Deemter curves should be measured by varying the linear velocity at constant pressure and temperature. In fixed-restrictor systems, variation of the linear velocity is possible only by replacing the flow restrictor. With the variable-flow system, this problem can be overcome.

Figure 8 shows Van Deemter curves for hexatriacontane $\left(n-\mathrm{C}_{36}\right.$ ) and cyclohexane measured using the dual-restrictor system. No peak asymmetry due to dead volumes in the $T$ piece was observed.

Figure 8 shows a reduced plate height of 45 for $n-C_{36}$ at a linear velocity of $7 \mathrm{~cm} / \mathrm{s}$, resulting in only about 210 plates/ $\mathrm{m}$. In contrast, a reduced plate height of less than 5 was observed for cyclohexane at the same velocity. For this component, a reduced plate height close to 1 was found at a more commonly employed velocity of $2 \mathrm{~cm} / \mathrm{s}$. This results in almost 10,000 plates $/ \mathrm{m}$. The large difference in the reduced plate heights is due to the lower diffusion coefficient for $n-\mathrm{C}_{36}$ in carbon dioxide than for cyclohexane. Furthermore, the capacity factor of cyclohexane is much lower than for $n-C_{36}$. Table I lists the capacity factors and the experimentally observed and calculated diffusion coefficients for cyclohexane and $n$ $\mathrm{C}_{36}$. Experimental diffusion coefficients were calculated from the slope of the $H u$ vs. $u^{2}$ plots. Extracolumn contributions to peak broadening were neglected. The column pressure drop at the highest linear velocity was calculated to be less than 1 bar. Calculated diffusion coefficients were obtained from the Wilke-Chang equation (16).

As the values in Table I indicate, fairly good agreement exists between the experimental and calculated diffusion coefficients. Hence, Table I indicates that it is possible to measure Van Deemter curves and diffusion coefficients with the system described in this work. The deviation between the calculated and the experimental $D_{\mathrm{m}}$ values for cyclohexane at 238 bar may be due to the inaccuracy of the capacity factor, which was close to zero.

\section{CONCLUSIONS}

1. The system presented in this work allows independent variation of column pressure and flow rate in capillary SFC. The instrumentation is relatively simple.

2. The mass flow rate through linear restrictors could be described accurately by simple model equations. An equation

Table I. Measured and calculated diffusion coefficients of cyclohexane and $n-C_{36}$ in carbon dioxide at $120^{\circ} \mathrm{C}$.

\begin{tabular}{lcccc}
\hline Component & $P($ bar $)$ & $k$ & $D_{\mathrm{m}}^{\exp }\left[\mathrm{cm}^{2} / \mathrm{s}\right]$ & $D_{\mathrm{m}}^{\mathrm{calc}}\left[\mathrm{cm}^{2} / \mathrm{s}\right]$ \\
$n-\mathrm{C}_{36}$ & 238 & 1.79 & $8.7 \cdot 10^{-5}$ & $9.0 \cdot 10^{-5}$ \\
cyclohexane & 238 & 0.04 & $1.9 \cdot 10^{-4}$ & $2.9 \cdot 10^{-4}$ \\
cyclohexane & 154 & 0.08 & $4.1 \cdot 10^{-4}$ & $4.1 \cdot 10^{-4}$ \\
cyclohexane & 100 & 0.19 & $5.5 \cdot 10^{-4}$ & $5.0 \cdot 10^{-4}$ \\
\hline
\end{tabular}


can be derived to quantify the column flow rate in the dualrestrictor system.

3. In comparison with a fixed-restrictor system, the flowcontrolled system shows better performance. Improved resolution is observed in the latter part of a pressure-programmed analysis.

4. For quantitative analysis, it is necessary to calibrate the system because the fraction of the effluent that is fed to the detector changes during the analysis.

5. The system allows the convenient measurement of Van Deemter curves and diffusion coefficients in open-tubular SFC. Diffusion coefficients were found to be in good agreement with the values calculated according to the Wilke-Chang equation.

\section{REFERENCES}

1. P.A. Peaden, J.C. Fjeldsted, M.L. Lee, S.R. Springston, and M. Novotny, Anal. Chem. 54, 1090 (1982).

2. T. Greibrokk, B.E. Berg, A.L. Blilie, J. Doehl, A. Farbrot, and E. Lundanes, J. Chromatogr. 394, 429 (1987).
3. E. Klesper and F.P. Schmitz, J. Chromatogr. 402, 1 (1987).

4. F.P. Schmitz, B. Gemmel, D. Leyendecker, and D. Leyendecker, $H R C \& C C 11,339$ (1988).

5. T. Takeuchi, K. Ohta, and D. Ishii, Chromatographia 25, 125 (1988).

6. S.R. Springston, P. David, J. Steger, and M. Novotny, Anal. Chem. 58, 997 (1986).

7. J.C. Fjeldsted, W.P. Jackson, P.A. Peaden, and M.L. Lee, J. Chromatogr. Sci. 21, 222 (1983).

8. Y. Hirata, F. Nakata, and M. Kawasaki, $H R C \& C C$ 9, 633 (1986).

9. Y. Hirata and F. Nakata, Chromatographia 21, 627 (1986).

10. D.E. Raynie, K.E. Markides, M.L. Lee, and S.R. Goates, Anal. Chem. 61, 1178 (1989).

11. R.W. Bally and C.A. Cramers, $H R C \& C C$ 9, 626 (1986).

12. R.D. Smith, J.L. Fulton, R.C. Petersen, A.J. Kopriva, and B.W. Wright, Anal. Chem. 58, 2057 (1986).

13. S. Angus, B. Armstrong, and K.M. de Reuck, Carbon Dioxide, International Thermodynamic Tables of the Fluid State-3 (Pergamon Press, Oxford, England, 1976) p. 44.

14. R.D. Smith, E.G. Chapman, and B.W. Wright, Anal. Chem. 57, 2829 (1985).

15. R. Feist and G.M. Schneider, Sep. Sci. Technol. 17(1), 261 (1982).

16. R.C. Reid, J.M. Prausnitz, and T.K. Sherwood, The Properties of Gases and Liquids, 3d ed. (McGraw-Hill, New York, 1977), p. 544. 